\title{
Evaluation of MPEG-4 Video Streaming Over Multi-hop Cellular Networks
}

\author{
Jinglong Zhou \\ Delft University of Technology, \\ Mekelweg 4, 2600 GA Delft, \\ The Netherlands \\ J.L.Zhou@tudelft.nl
}

\author{
Anthony Lo \\ Delft University of Technology, \\ Mekelweg 4, 2600 GA Delft, \\ The Netherlands \\ A.C.C.Lo@tudelft.nl
}

\author{
Ignas Niemegeers \\ Delft University of Technology, \\ Mekelweg 4, 2600 GA Delft, \\ The Netherlands \\ I.G.M.M.Niemegeers@tudelft.nl
}

\begin{abstract}
Next generation communication networks will comprise third generation (3G) cellular and multi-hop ad hoc networks. MPEG-4 video streaming can be a killer application for this novel multihop cellular network. The paper has evaluated the end-to-end performance of streaming MPEG-4 video over a multi-hop cellular network that comprises the High-speed Downlink Packet Access (HSDPA), Universal Mobile Telecommunication System (UMTS) and the IEEE 802.11 ad hoc networks. The factors, which can affect the end-to-end performance of MPEG-4 video transmission, are analyzed using simulation. For the same configurations, the simulation results reveal that different MPEG-4 video contents can cause different performance for HSDPA. The transmission strategies used in HSDPA and UMTS also results in performance differences. Moreover, the number of hops, error rate in the 802.11 multi-hop network and traffic contentions level have a strong impact on the end-to-end MPEG-4 video streaming performance. Our results can be used for gateway selection, resource reservation and QoS provision in the novel multi-hop cellular network.
\end{abstract}

\section{Categories and Subject Descriptors}

C.2.1 [Network Architecture and Design]: Wireless communication

\section{General Terms}

Algorithms, Performance

\section{Keywords}

UMTS/HSDPA, IEEE802.11, multi-hop cellular network, MPEG4

\section{INTRODUCTION}

Widely deployed third generation (3G) cellular networks such as the Universal Mobile Telecommunications System (UMTS) technology supports multimedia applications. High Speed Downlink Packet Access (HSDPA) [2] evolves from the UMTS radio interfaces on the downlink with a peak data rate up to $14.4 \mathrm{Mbps}$. HS-

Permission to make digital or hard copies of all or part of this work for personal or classroom use is granted without fee provided that copies are not made or distributed for profit or commercial advantage and that copies bear this notice and the full citation on the first page. To copy otherwise, to republish, to post on servers or to redistribute to lists, requires prior specific permission and/or a fee.

SIMUTools 2010 March 15-19, Torremolinos, Malaga, Spain

Copyright 2010 ICST, ISBN 78-963-9799-87-5.
DPA is sometimes referred to as $3.5 \mathrm{G}$. The IEEE 802.11 technology enables short range high speed connection in two modes, infrastructure and ad hoc mode.

Both technologies have its own advantages and disadvantages. The $3 \mathrm{G}$ cellular networks can provide wide range coverage and high mobility support but their data rates are still limited. IEEE 802.11 can support high data rates up to $200 \mathrm{Mbps}$ for IEEE $802.11 \mathrm{n}$ and popular among portable electronic devices, such as laptops and PDAs. Integrating those two technologies can provide Internet access for those IEEE 802.11 enabled devices that do not have 3G cellular network interface. Further, the integrated network can leverage the advantages of these two technologies, e.g., load balancing for $3 \mathrm{G}$ cellular network, relaying traffic for the device that suffers from bad channel conditions. A number of previous works proposed architectures for integrating the $3 \mathrm{G}$ cellular networks and ad hoc networks. [10] proposed a hybrid cellular and ad hoc network architecture namely, UCAN, to increase cell throughput without sacrificing fairness. The proposed architecture required every mobile station to have both cellular and IEEE 802.11 ad hoc interfaces. [5] described an integrated ad hoc and cellular network architecture whereby base stations are involved in coordinating peerto-peer communications in order to increase cell throughput and coverage. However, none of them discussed the end-to-end performance over this new integrated network.

Video streaming is a potential application of the cellular ad hoc network. MPEG-4 [7] is one of video streaming encoding technologies which generate low bit rate video streams, therefore it is suitable for bandwidth limited wireless networks. The special traffic characteristics and strictly delay constraint of video traffic have different requirements for $3 \mathrm{G}$ cellular network compared to other traffic, e.g., Transmission Control Protocol (TCP). [3] and [8] evaluated the performance of MPEG-4 over UMTS networks via mathematical models and simulations, respectively. However, the endto-end performance evaluation of the MPEG-4 video source over multi-hop cellular network with HSDPA channel is still missing. The different retransmission mechanisms introduced by HSDPA can have impact on the MPEG-4 video transmission. Compared to [8], we used advanced UMTS technology, HSDPA. More important, this cellular network is integrated with IEEE 802.11 network in the simulator. The route selection in this new network can bring in performance enhancement. This simulator allows us to research the trade off in route selection between bad cellular channel and IEEE 802.11 channels using validity simulation assumptions, e.g, a bad HSDPA channel or a good HSDPA channel combined with 802.11 relaying, which one to choose.

To the best of our knowledge, this is the first paper to research the end-to-end performance of MPEG-4 over the multi-hop cellular ad hoc network with HSDPA channel model. A tool-set for 


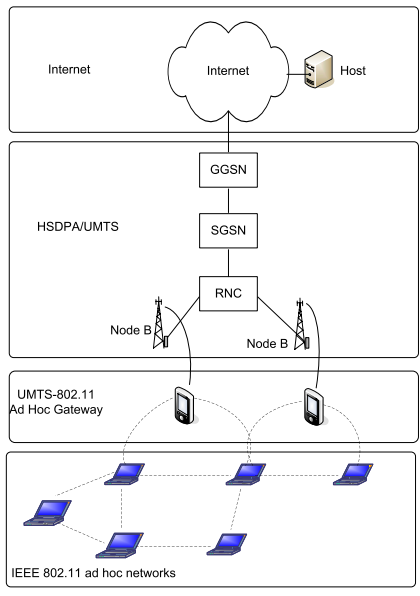

Figure 1: Multi-hop cellular architecture overview.

evaluating and performance optimization of MPEG-4 traffic over integrated network is introduced. The rest of the paper is organized as follow; Section 2 introduce the background technology, our simulation model is described in Section 3 and in Section 4 we present the simulation result, the paper is concluded in Section 5.

\section{MULTI-HOP CELLULAR NETWORK AND MPEG-4}

In this section, we give an overview of multi-hop cellular networks and MPEG-4.

\subsection{System Architecture}

Fig. 1 depicts the architecture of the multi-hop cellular network. The Internet is connected to a host and the UMTS network. A node which has both UMTS and IEEE 802.11 interfaces acts as a gateway between the UMTS network and the ad hoc network. The gateway includes protocols for gateway-discovery, gateway selection, address allocation, routing, session negotiation, which were addressed in [9].

To be able to realize our newly integrated network, we proposed the following network protocol stacks as depicted in Fig. 2. The right part of the stacks are the UMTS core network, which consists of Node B, Radio Network Controller (RNC), Serving GPRS Support Node (SGSN) and Gateway GPRS Support Node (GGSN). The UMTS/HSDPA network and the IEEE 802.11 ad hoc networks are interconnected via an HSDPA-802.11 Ad-hoc Gateway, which has two different network interfaces as depicted in Fig. 2. The HSDPA-802.11 ad hoc gateway is refered to as mobile gateway in the rest of the paper. To enable the mobile nodes in ad hoc network to find and select the mobile gateway, gateway discovery protocol is needed over the IP layer in the mobile gateway. The mobile gateway implements the ad hoc routing protocol to relay the packet for ad hoc network.

\subsection{MPEG-4}

MPEG-4 is collection of methods defining compression of audio and visual (AV) digital data and standardized by ISO/IEC. The standard defines tools to create, represent and distribute individual audiovisual objects, both natural and synthetic. MPEG-4 provides the advanced video coding (AVC) compression process that consumes smaller bandwidth. As a result, it is likely to become the preferred approach for next-generation digital image distribution.

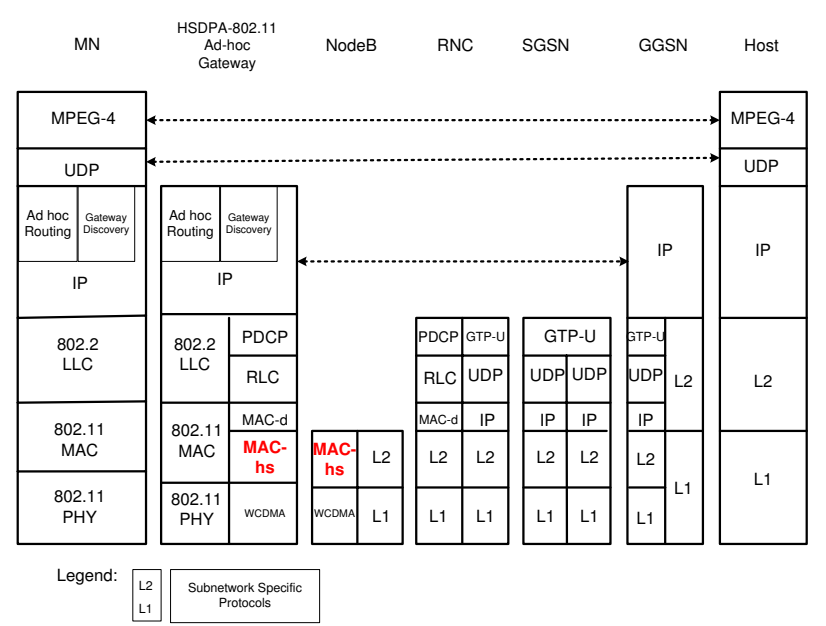

Figure 2: Multi-hop cellular protocol Stacks.

An MPEG-4 encoder generates three types of frames, Intra-frames (I-frames), Predictive frames (P-frames) and Bi-directional frames (B-frames). The P, B frames have higher compression and I frame can be encoded and decoded faster.

\section{SIMULATION MODELS}

\subsection{Integrated Network Module in ns-2}

EURANE [1] was extended to support the multi-hop networks and generate the UMTS/HSDPA channel input trace file to realize the end-to-end IP packet transmission over UMTS/HSDPA. The detailed description of this integrated network can be found in [12].

The UMTS and HSDPA have two major differences. Firstly, the UMTS system uses power adaption to maintain a fixed error rate. In the simulation, the packet error rate was set to $0 \%-50 \%$ on the dedicated channels. However, HSDPA system uses a shared channel that has constant transmission power. Thus, users can experience different channel conditions. In the simulation, the HSDPA used a pre-generated trace file that models channel behavior [12]. To compensate for the random effect introduced by the channel model, all simulations with HSDPA channel were repeated 10 times with different trace files and the average result was used. Using different distances between Node B and UE (User Equipment), eight different types of HSDPA channel quality files $(0-700 \mathrm{~m})$ were generated. The average Signal to Noise Ratio (SNR) value was used for representing the HSDPA channel quality levels. Secondly, the method of retransmitting lost frames. UMTS system uses the RLC layer for retransmission, which is between the RNC node and the UE. HSDPA uses MAC for retransmission which is between Node B and UE. The retransmission scheme of HSDPA is faster due to smaller Transmission Time Interval (TTI).

\subsection{MPEG-4 Video Evaluation Tool-set}

EvalVid [7] is a tool-set for evaluating video quality transmitted over a simulated communication network. [6] further extended this tool-set to include ns-2. We integrated EURANE into the tool-set. The integrated environment is depicted in Fig. 3. The raw video with YUV format is encoded into the MPEG-4 format with a certain coding rate. The raw video source used in the simulation is the Common Intermediate Format (CIF) format $(352 \times 288$ pixels $)$. The MPEG-4 encoder supports different coding rates according 


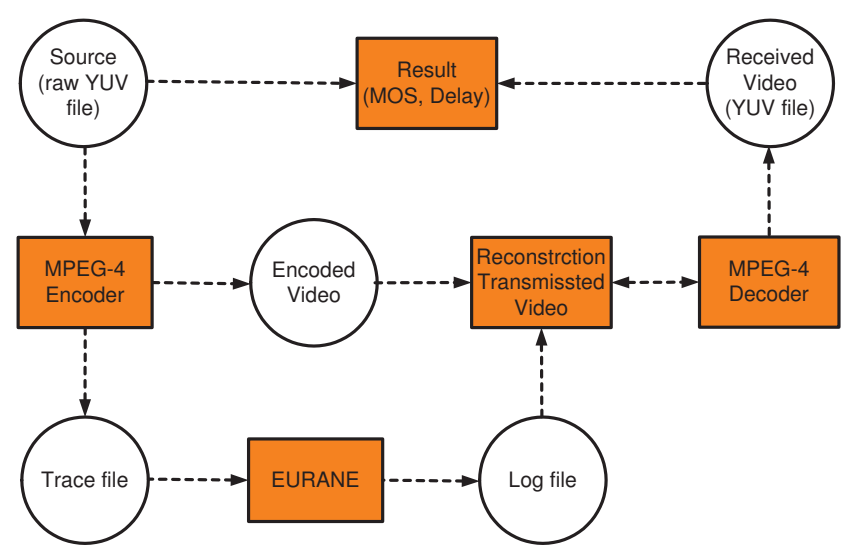

Figure 3: MPEG-4 video stream evaluation tool-set.

to user requirement. The encoded MPEG-4 video is segmented into UDP packets based on maximum segmentation packet size and captured into a trace file, which contains the information of transmitted packet departure time. The trace file is fed into the EURANE traffic generator agent which in turn is segmented and encapsulated in the UDP datagrams. The received video frames in the simulation is recorded by the simulator into the Log file. The program reconstructs the video using the compressed video and the received log file. This transmitted video is decoded again and is compared to original raw video frames.

Two metrics, namely, the end-to-end delay of video packets and Mean Opinion Score (MOS) were used to evaluate the performance. MOS is the human impression of the video quality, which is given on scale from 1 to 5 [8]. During the simulation, erroneous video packet due to impairment of cellular radio channels will be recovered by the link layer. We assume that IEEE $802.11 \mathrm{~b}$ links are ideal and no packets will be lost. MPEG-4 video streaming is a delay constraint application, packets arrived later than the buffer time will be dropped. This leads to quality degradation, and thus, lower MOS.

\subsection{Simulation Setup}

The simulations were carried out using the topology shown in Fig. 4. We used the SGSN as our MPEG-4 traffic source. In the ad hoc network, a chain topology was used to connect the 802.11 nodes, the MPEG-4 receiver can be the mobile gateway, MN1 and MN2, which corresponded to 0-hop, 1-hop and 2-hop. The configuration of wired link is shown in Fig. 4. The configuration of the wireless link, MPEG-4 video streaming parameters are in Table. 1. In the simulation, the IEEE 802.11b's highest data rate was used, this is because we assume our applications are outdoor and long distance transmission between IEEE 802.11 nodes $(130 \mathrm{~m}$ in simulation), some literature indicated that $802.11 \mathrm{~g}$ radio performance badly in those conditions [4].

\subsection{Video contents}

The video source we are using are the "waterfall" and "flower" scenarios shown in Fig. 5. The packet distribution of this scenario is depicted at Fig. 5(c) and Fig. 5(d). The raw video rate of "waterfall" and "flower" is $3 \mathrm{Mbps}$ and $4 \mathrm{Mbps}$, respectively. We coded them into $1 \mathrm{Mbps} \mathrm{mp} 4$ file for network transmission.

\section{SIMULATION RESULTS}

The factors, which can influence the video quality, are transmis-

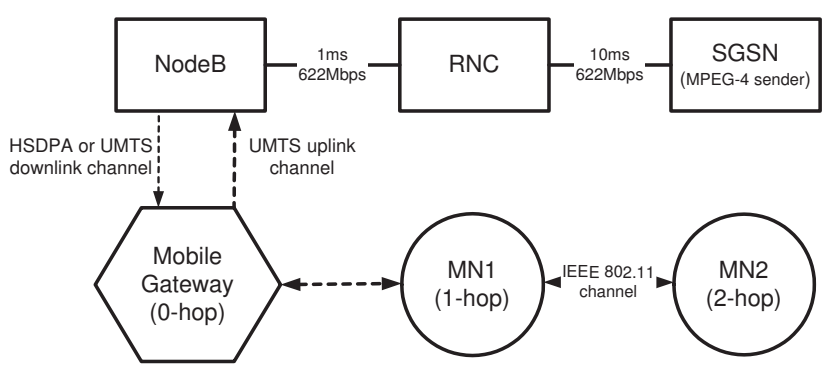

Figure 4: Simulation Topology.

Table 1: Simulation parameters

\begin{tabular}{c|c}
\hline HSDPA & Values \\
\hline Distance from NodeB & $0-700 \mathrm{~m}$ \\
Path loss component (n) & 3.52 \\
Mobile gateway speed & $3 \mathrm{~km} / \mathrm{h}$ \\
Mobile gateway move pattern & circle \\
Standard deviation in shadow fading (N) & $8 \mathrm{~dB}$ \\
Maximum buffer size at NodeB & $1000 \mathrm{Kbytes}$ \\
UPlink TTI & $10 \mathrm{~ms}$ \\
Downlink TTI & $2 \mathrm{~ms}$ \\
Uplink bandwidth & $384 \mathrm{Kbps}$ \\
\hline UMTS & Values \\
\hline UPlink TTI & $10 \mathrm{~ms}$ \\
Downlink TTI & $10 \mathrm{~ms}$ \\
Uplink bandwidth & $384 \mathrm{Kbps}$ \\
Downlink bandwidth & 384 or $2084 \mathrm{Kbps}$ \\
\hline IEEE 802.11 & Values \\
\hline IEEE 802.11 bandwidth & $11 \mathrm{Mbps}$ \\
RTS/CTS Threshold & $3000 \mathrm{Bytes}$ \\
\hline MPEG-4 & Values \\
\hline Video Coding rate & $1 \mathrm{Mbps}$ \\
Video buffer time & $1 \mathrm{~s}$ \\
Number of frames per second & 25 \\
Maximum segmentation packet size & $1000 \mathrm{Bytes}$ \\
\hline
\end{tabular}

sion strategies, network topology, channel quality and traffic contention levels are evaluated in this section.

\subsection{IEEE 802.11 error free channel}

\subsubsection{Impact of Video Sources}

Each video source will have different packet distribution. The video with high temporal information produces larger $\mathrm{P}$ and $\mathrm{B}$ frames and the video with more stationary contents produce larger I frames. In the simulation, we picked two different video sources which are shown in Fig. 5(a) and Fig. 5(b). We call these two video streams "flower" and "waterfall" scenario, respectively. Fig. 5(c) and Fig. 5(d) depict the segmentation packet sizes with frame numbers of these two video streams. We can see that the "flower" video stream has larger P, B frames and "waterfall" video stream has larger I frames at the start of the video stream.

We use 1-hop scenario with HSDPA channel to evaluate the performance difference caused by different video sources. In Fig. 6, we depict the average MOS value with different channel conditions. We can see that the average MOS value for the flower scenario begins to decrease when the average SNR decreases to $5.43 \mathrm{~dB}$. The average MOS value further drops to 2.8 , when the channel quality becomes worse. The MOS value drop to 1 when the channel 


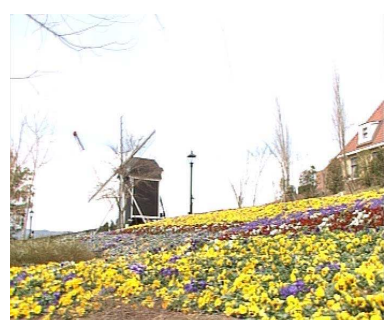

(a)

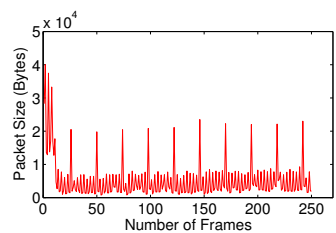

(c)

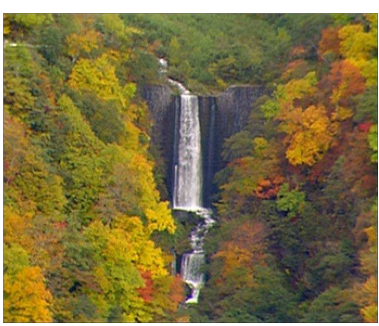

(b)

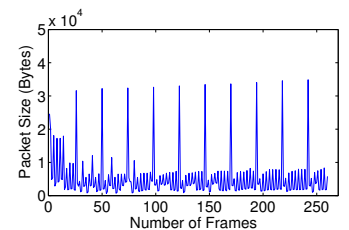

(d)
Figure 5: Different video source comparison: (a) flower video, (b) waterfall video, (c) flower video, segmentation packets size vs time, (d) waterfall video, segmentation packets size vs time.

quality is $0.18 \mathrm{~dB}$. However, for the waterfall scenario, the MOS value only begins to decrease when the average SNR is $2.58 \mathrm{~dB}$. The MOS value of waterfall video is always higher than the flower video even when the channel quality is the same. Video source packet distribution deviation caused the difference.

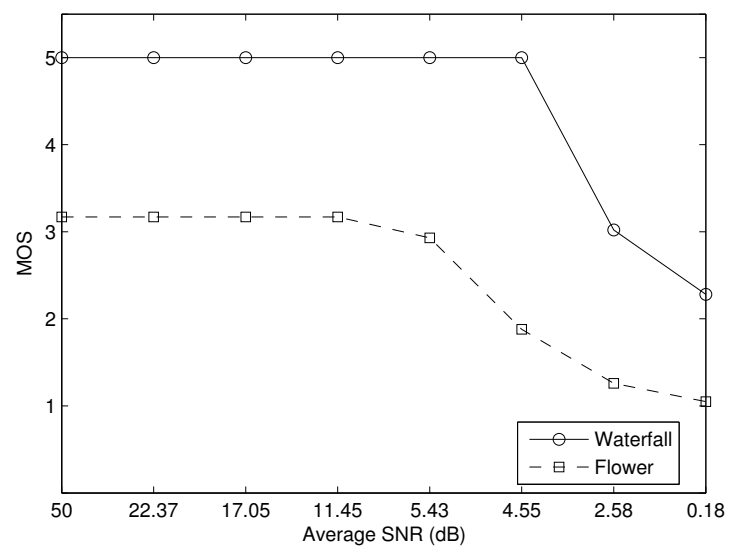

Figure 6: Different MPEG-4 video sources over HSDPA channel: MOS compare.

The end-to-end delay cumulative distribution function shows the network performance for delivery of a certain video source. We depict the delay when the HSDPA channel have an average SNR of $22.37 \mathrm{~dB}$. Fig. 7 depicts the end-to-end delay cumulative distribution function of the two video streams. In this network setting, all packets have a delay less than the buffer time (1s). We can observe that about $70 \%$ the packets of the two video streams have the same delay distribution. For the other $30 \%$, the packets from video source flower have larger delay than the packets from the waterfall video source.

As shown in Fig. 6, the MOS of flower is lower than waterfall even under ideal HSDPA channel condtions. This is due to lossy MPEG-4 encoding. The flower video source has consecutively sev- eral big packets at the beginning of the video. Thus, at beginning of video transmission the delay for flower scenario increases and reaches the maximum $0.45 \mathrm{~s}$, then decrease to normal level again. The flower video use larger P, B frames which result in more transmission delay in HSDPA MAC layer. The result shows that video source different can cause large performance difference under the same network conditions.

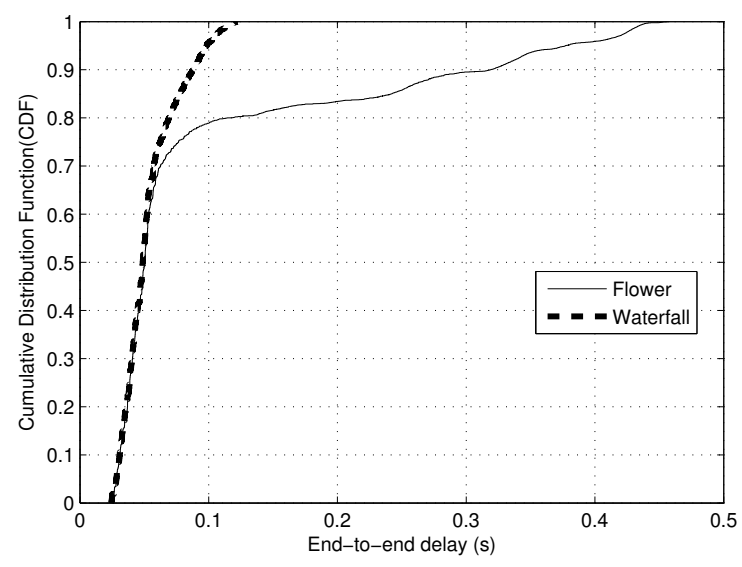

Figure 7: Different MPEG-4 video sources over HSDPA channel: Delay compare.

\subsubsection{Impact of HSDPA and UMTS Transmission Strat- egy}

As described in Section 3.1, HSDPA and UMTS system use different retransmission strategies. The UMTS uses the RLC layer to retransmit lost frames. In the RLC layer, two timers, namely, the status prohibit timer and poll timer, are used to control the retransmission process. The status prohibit timer and poll timer located at both UE and RNC dictate when the retransmission will take place. Larger timer value will slow down the retransmission process while smaller timer may trigger unnecessary retransmission which costs bandwidth.

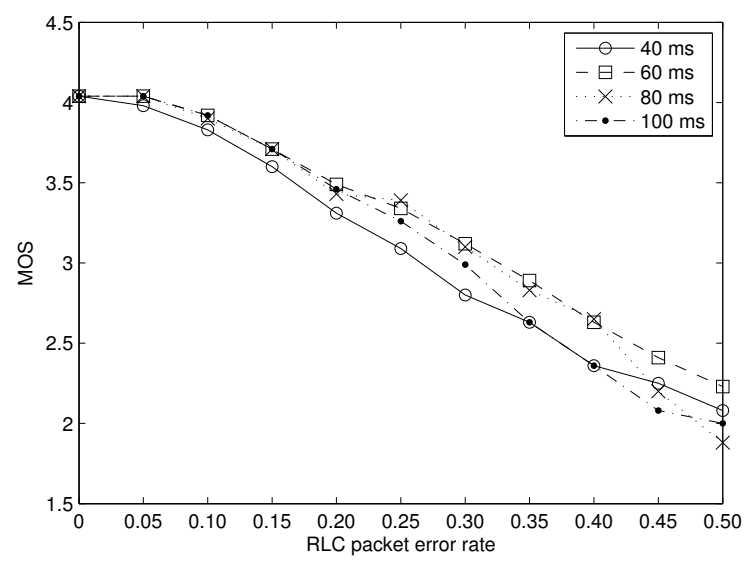

Figure 8: MPEG-4 video over UMTS channel with different retransmission timer values.

We carried out experiment with UMTS channel with the downlink bandwidth $384 \mathrm{Kbps}$. The performance comparison of differ- 
ent RLC timer values is depicted in Fig. 8. We used four different timer values and the poll timer and status prohibit timer were set to be the same. It is obvious that too large $(100 \mathrm{~ms})$ or small $(40 \mathrm{~ms})$ will not result in optimal performance. It is interesting to see that no single timer value achieved the best performance for all channel conditions. Generally the $60 \mathrm{~ms}$ achieved the best performance for most of the scenarios. A smaller value $(40 \mathrm{~ms})$ perform slightly worse than larger value when the channel quality or packet error rate is from $0 \%$ to $35 \%$, which indicates that small timer values result in redundant retransmissions that decrease the performance. When the channel becomes very bad (packet error rate of $35 \%$ to $50 \%$ ), smaller values $(40 \mathrm{~ms})$ outperform larger ones due to faster retransmission. The result indicates that for the UMTS channel, the optimal timer value for video transmission should be adaptive based on channel conditions.

The HSDPA system uses the fixed interval to retransmit the lost MAC frames, no timer is employed. However, the variance of channel quality can have an impact on video transmission quality. In Fig. 9, we show the end-to-end delay cumulative distribution function of video packet delivery for HSDPA transmission with different channel conditions. We can see that, when the average SNR value is higher than $17.05 \mathrm{~dB}$, the end-to-end delay is always within $150 \mathrm{~ms}$. When the quality of the channel deteriorates, end-to-end delay increases.

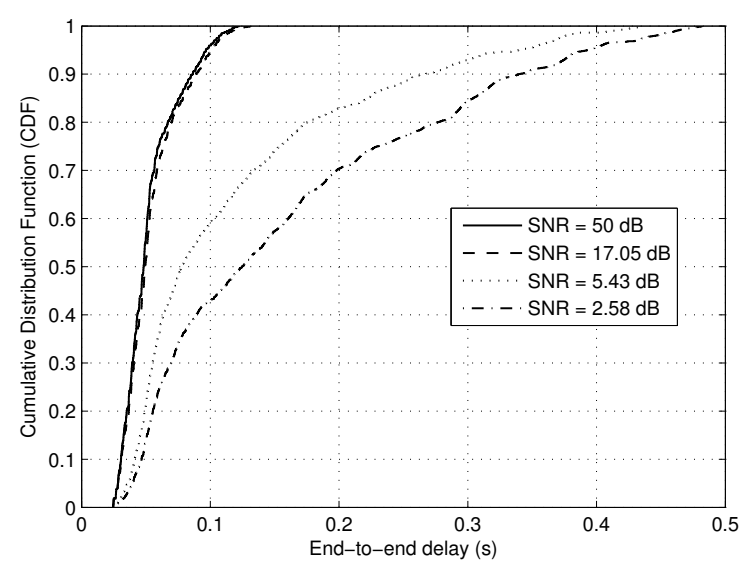

Figure 9: MPEG-4 video over HSDPA channel with different channel conditions.

Now, we compare the performance of MPEG-4 over these two transmission strategies. For a fair comparison, we select a set of parameters for both HSDPA and UMTS, which gives the same maximum TCP throughput. For UMTS, the settings for the downlink channel were $2048 \mathrm{Kbps}$ and a fix packet error rate of 5\%. For HSDPA, the setting is the average SNR which was 2.58 dB. Fig. 10 plots the end-to-end delay of video packets for HSDPA and UMTS. We can observe that the delay distribution is different, the delay in UMTS system has less deviation. For HSDPA, $85 \%$ of the packets have lower delays compared to UMTS due to the faster retransmission mechanism. However, the other $15 \%$ of the packets have even longer delays than UMTS. This result is due to the fact that UMTS has fixed error rates which leads to more stable performance, while HSDPA channel variance has strong effect on the end-to-end delay.

\subsubsection{Impact of the Network Configuration in Multi- hop Cellular Network}

Different configurations will result in different minimum band-

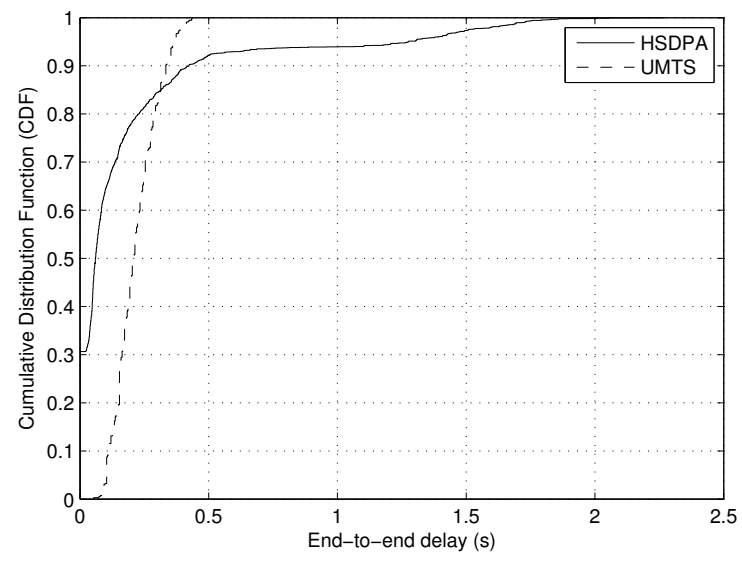

Figure 10: Delay compare between UMTS and HSDPA channel for MPEG-4 video stream.

width for cellular and ad hoc network. The integrated multi-hop cellular network will have different network bottleneck under different configurations [12]. As we described in Section 3.2, if the encoded MPEG-4 video bit rate is higher than this bottleneck, packets delay may be higher than the buffer level and cause the MOS value to decrease.

Traffic contention and number of hops will impact the video quality. In this section, we have two different scenarios, one is with different number of hops extension in multi-hop ad hoc network, the other scenario is we set two flows, one is MPEG-4 video traffic with $1 \mathrm{Mbps}$ bit rate and the other one is Constant Bit Rate (CBR) traffic in the shared channels. The CBR traffic is called contention traffic. The HSDPA channel is used. The factors' impact on the MOS value is depicted in Fig. 11 and Fig. 12 respectively. As discussed in Section 3, we used 0, 1 and 2-hop topology in the multi-hop network for Fig. 12 and three different contention traffic levels with 1-hop topology are used for Fig. 11. It is obvious to see that the MOS value is the same for the 0 and 1-hop scenario, and MOS value in 2-hop begin to decrease when the HSDPA channel is bad, which indicates that with more hops extension in the multihop ad hoc network, the increased end-to-end delay can decrease the MPEG-4 video stream quality when HSDPA channel quality is bad.

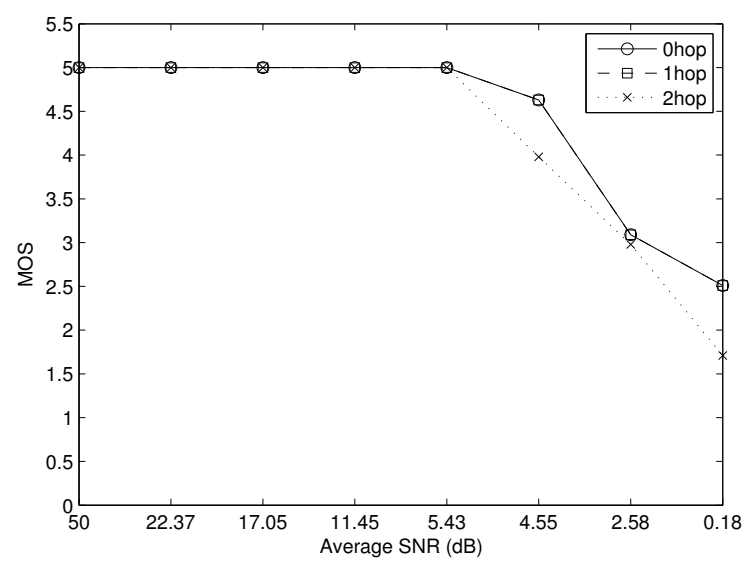

Figure 11: Number of hops extension. 
For the total traffic, the MPEG-4 bit rate is $1 \mathrm{Mbps}$ and contention traffic load is $1 \mathrm{Mbps}$. Meanwhile, the maximum HSDPA channel bandwidth is $3.36 \mathrm{Mbps}$. We can see that with different contention traffic intensity, the MOS value decrease differently when the channel's SNR decrease. When the average SNR is $5.43 \mathrm{~dB}$, the MOS value with $500 \mathrm{Kbps}$ have a MOS value 4.52 and $1000 \mathrm{Kbps}$ scenario only got 4.3. When the average SNR is $4.55 \mathrm{~dB}$, the average MOS value drops to 3 which means the video quality is very bad and unacceptable. The simulation results demonstrate the QoS provision should be provided based on precise knowledge of the network configuration and status. When the channel quality is bad (average SNR lower than $2.58 \mathrm{~dB}$ ), the video transmission performance also variance a lot based on our result.

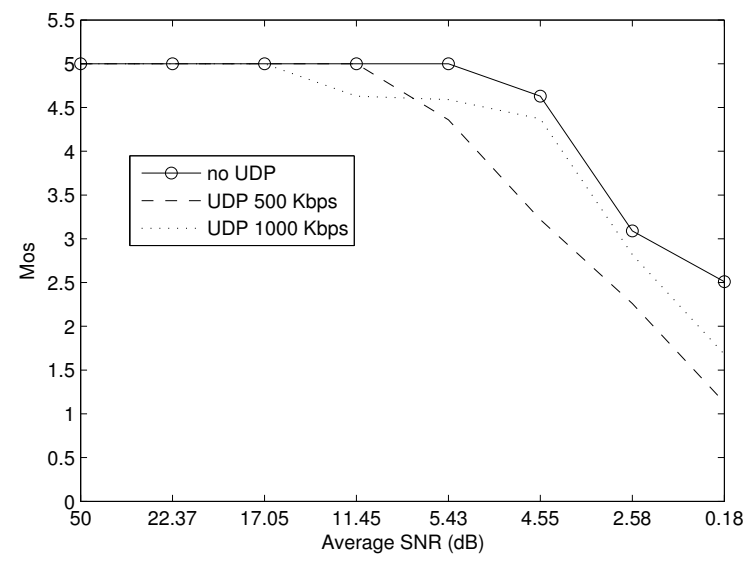

Figure 12: Different contention traffic levels.

The same as in Section 4.1.2, we still use the end-to-end delay result when the HSDPA channel have an average $\mathrm{SNR}=22.37 \mathrm{~dB}$. We used 5 different contention traffic levels in the simulation and plot the delay in Fig. 13. We can see that when there is no contending traffic and with $500 \mathrm{Kbps} \mathrm{CBR}$ contending traffic, the performance is similar. When there is higher contention traffic passing the channel, the end-to-end delay increase much more and can reach up to $0.3 \mathrm{~s}$.

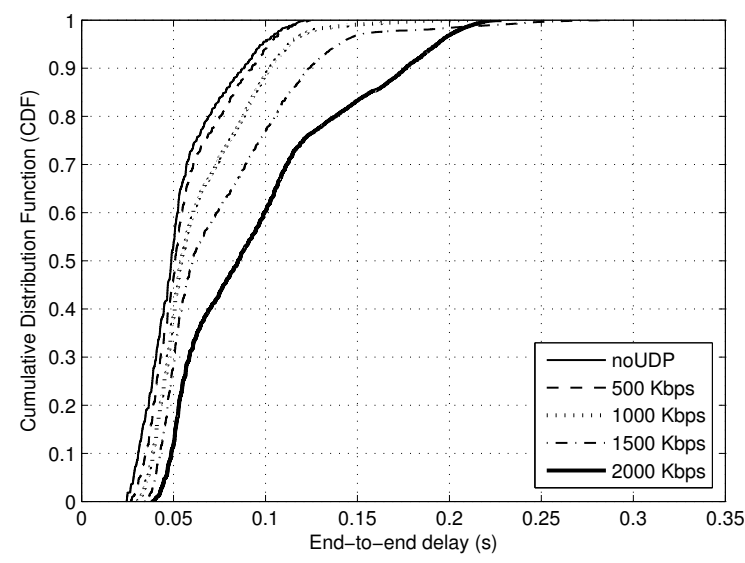

Figure 13: End-to-end packet delay with multiple flows.

\subsection{Erroneous IEEE 802.11 channels}

With the retransmission scheme in our cellular network simulation model, there will be no UDP packet loss during the transmission in the cellular network. In previous section, we assume there is no error in IEEE 802.11 links. Therefore, the MOS value decrease is only caused by the delayed packets. The impact of IEEE 802.11 links to the end-to-end performance is not obvious due to its short delay. In this section, we also set uniform distributed error in the IEEE 802.11 channel. We used four error rates in our simulation, $10 \%, 20 \%, 30 \%$ and $50 \%$. For the 802.11 channel, we set the maximum number of retransmission for data packet to be 7 .

We first use the one hop topology in the 802.11 channel, the same as in Fig. 9. We use different HSDPA channel traces with different average SNR value. The MOS value is shown in Fig. 14. We can see that for all the scenarios, the MOS value decrease when the HSDPA channel have lower average SNR. When the error rate in IEEE 802.11 increases, the MOS value decreases dramatically. For 50\% error rate, the MOS value decreases to 1 which means the video can not be seen at all. It is interesting to notice that only until 20 percent error, the video quality start to decrease when the HSDPA channel is still in good quality $(S N R>4.55 \mathrm{~dB})$. In the situation which HSDPA has very low average SNR, $(S N R<4.55 \mathrm{~dB})$, the HSDPA channel causes the MOS value to be further decreased. This MOS value result indicates that bottleneck effect (see Fig.6 in [12]) will be more obvious when there is error in IEEE 802.11 channel. The end-to-end video quality can be impacted fluctuated easily due to the channel quality variance in cellular network or ad hoc network.

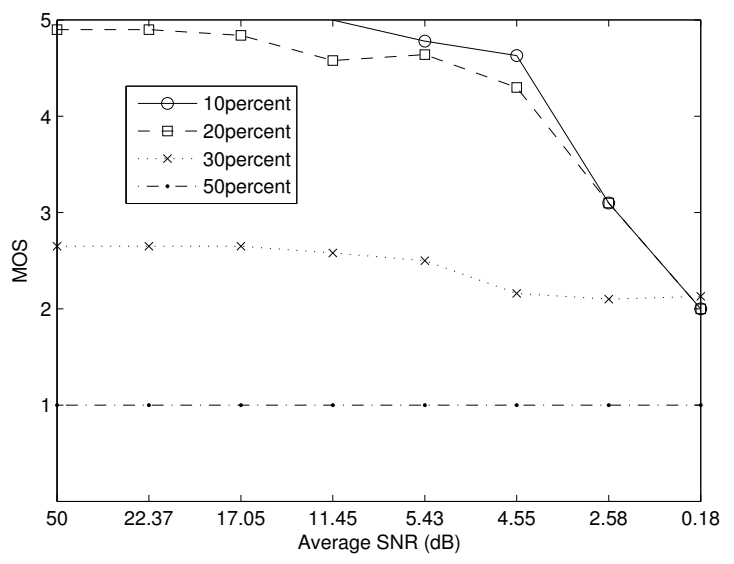

Figure 14: The MOS value decrease in different error rate.

It is also interesting to look at the end-to-end delay in Fig. 15. When the error rate is higher, lost of video packets are lost. In this figure, we only plot only the received packets average delay. We can see that with higher error rates in IEEE 802.11, the average delay even decreases.

We also did the simulations with different number of hops extension in ad hoc network with the same IEEE 802.11 error rate, all the simulations use the same HSDPA channel trace which have an average SNR $50 \mathrm{~dB}$. Based on Fig. 16, we can see that when the number of hops increases, the MOS value further decreases dramatically. When the error is higher than 20 percent, the MOS value for the 2-hop scenario is lower than 1.5, which means the 2-hop topology can not be used at all when the error rate is higher than 20 percent. 


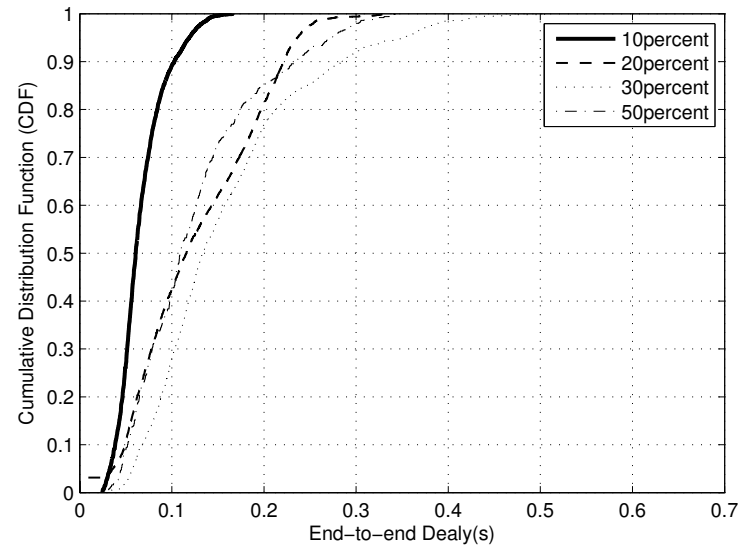

Figure 15: The delay distribution in different error rate.

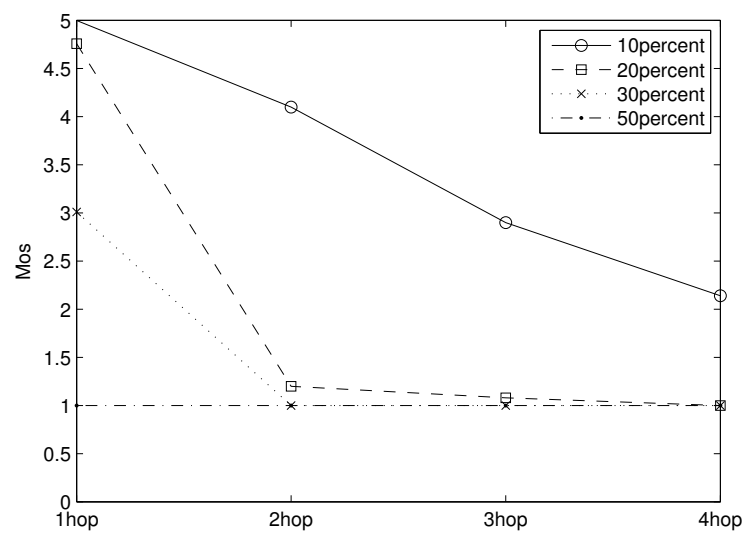

Figure 16: The MOS value decrease in multi-hop network.

\subsection{Discussion}

The simulation result shown in previous two sections indicate that both networks, cellular and 802.11 ad hoc network can cause MPEG-4 video performance degradation in the receiver side. The cellular network use the ARQ mechanism to prevent packet loss but also result in longer delay for the video packet which causes the video quality decrease. The IEEE 802.11 MAC layer also have the retransmission attempts which can prevent parts of packet loss, but still can cause packet delay and reduce the MPEG-4 video quality due to packet loss. In the scenario which have several gateways for a large scale ad hoc network, the end users experience can be effected by both networks. In order to achieve the best end-to-end video quality, the mechanism for selecting the best gateway and relaying node need to be designed. The mechanism should consider the effect of delay in cellular network and packet loss in 802.11 ad hoc network, make the best combination during the route selection.

Our MPEG-4 simulation tool-set can be used for designing and evaluating this new mechanism. The cellular network uses the channel model based on the distance between user node and base station. The IEEE 802.11 can also use the distance to decide the channel quality. Based on the measurement result from [11], the average SNR for error rate of 0.5, 0.3, 0.2 and 0.1 is 20, 24, 26 and $28 \mathrm{~dB}$. The assumption that IEEE 802.11 radio link's SNR values
22.37, 17.05 etc correspond to $10,20 \%$ etc error rate is draw from the Packet Delivery Ratio (PDR) to SNR mapping got from our measurement experiment using our real IEEE 802.11 test-bed, with real IEEE 802.11 channels. The packet error rate for a certain channel is calculated by more than 1000 UDP packets and the SNR is calculated by the average SNR more than 100 probe packets (small UDP data packet, 2Mbps). For a certain time period (1s), we got one mapping points (one PDR and one SNR) and we gather those points for very long time to form a mapping graph show in figures in [11]. The NS-2 simulator can calculate the SNR between two nodes based on their distance based on channel model. We can use the SNR to PDR mapping achieved from measurement to predict the correspond error rate between these two ad hoc nodes. Therefore, we can achieve a quite realistic simulation environment and with our simulation tool-set, we can validate the performance of special gateway and route selection protocol which select the best relaying nodes and gateway combination for the MPEG-4 traffic.

\section{CONCLUSION}

From the simulation results, we conclude that for the novel multihop cellular network, high quality MPEG-4 video streaming with compressing bit rate of $1 \mathrm{Mbps}$ is feasible. Using an integrated MPEG-4 tool-set, we analyzed the impact of several factors on the MPEG-4 video streaming performance over the novel integrated multi-hop UMTS/HSDPA and IEEE 802.11 network. The simulation results demonstrate that the difference in UMTS/HSDPA radio transmission strategies have an impact on the end-to-end performance which requires different buffer sizes. Depending on number of hops away from the gateway, IEEE 802.11 error rate, cellular channel quality and contenting traffic levels, the end-users will have different MPEG-4 application quality experience, the link quality variance in both network will result in dynamic network bottleneck, which suggests that the selection of relaying node and gateway should consider those factors and dynamically made the selection, which is our future work.

\section{ACKNOWLEDGMENTS}

This work was supported by the Freeband PNP2008 research project. We would like also to express the appreciation for the suggestions of the anonymous reviewers.

\section{REFERENCES}

[1] Eurane, http://www.ti-wmc.nl/eurane/.

[2] HSDPA overall description (rel.5). 3GPP TS 25.308, 5.2.0, 2002/03.

[3] A. Alexiou, D. Antonellis, and C. Bouras. Adaptive and reliable video transmission over UMTS for enhanced performance. International Journal of Communication Systems, 1(1):65-81, 2007.

[4] G. Bianchi, F. Formisano, and D. Giustiniano. 802.11 b/g Link Level Measurements for an Outdoor Wireless Campus Network. In Proc. IEEE WoWMoM'06, pages 525-530, Washington, DC, June 2006.

[5] H. Hsieh and R. Sivakumar. On Using Peer-to-Peer Communication in Cellular Wireless Data Networks. IEEE Trans. Mobile Comput., pages 57-72, 2004.

[6] C. Ke, C. Lin, C. Shieh, and W. Hwang. A novel realistic simulation tool for video transmission over wireless network. In Proc. IEEE SUTC'06, Taichung, Taiwan, June 2006.

[7] J. Klaue, B. Rathke, and A. Wolisz. Evalvid - a framework for video transmission and quality evaluation. In Proc. 13th 
International Conference on Modelling Techniques and Tools for Computer Performance Evaluation, Urbana, Illinois, September 2003.

[8] A. Lo, G. Heijenk, and I. Niemegeers. Performance evaluation of MPEG-4 Video Streaming over UMTS Networks using an Integrated tool Environment. In Proc. SPECTS'05, Philadelphia, PA, July 2005.

[9] A. Lo, J. Zhou, and I. Niemegeers. Multi-hop Cellular Networks: Integrated IEEE 802.11 Ad hoc and Universal Mobile Telecommunications System (UMTS) Networks. In Proc. ERCIM eMobility Workshop 2008, pages 37-48, Tampere, Finland, May 2008.

[10] H. Luo, R. Ramjee, P. Sinha, L. Li, and S. Lu. UCAN: a unified cellular and ad-hoc network architecture. In Proc. ACM Mobicom'03, pages 353-367, San Diego, CA, September 2003.

[11] J. Zhou, C. Guo, P. Pawełczak, and I. G. Niemegeers. Adaptable link quality estimation for multi data rate communication networks. In Proc. IEEE VTC2009-Spring, Barcelona, Spain, Apr. 26-29, 2009.

[12] J. Zhou, A. Lo, Z. Liu, and I. Niemegeers. TCP Performance Evaluation Over Multi-hop Cellular Network: HSDPA and IEEE 802.11. In Proc. ISWCS'08, Reykjavik, Iceland, October 2008. 